Check for updates

Cite this: RSC Adv., 2021, 11, 5753

Received 21st August 2020

Accepted 21st January 2021

DOI: $10.1039 / \mathrm{dOra07194c}$

rsc.li/rsc-advances

\title{
Reply to the 'Comment on "Investigation of dielectric constants of water in a nano-confined pore"' by S. Mondal and B. Bagchi, RSC Adv., 2020, 10, DOI: 10.1039/DORA02726J
}

\author{
Haochen Zhu, (DD ab Han Hu, ${ }^{\text {ab }}$ Bo Hu, ab Wenzhi He, ${ }^{\text {ab Juwen Huang }}{ }^{\text {ab }}$ \\ and Guangming Li*ab
}

In our original work, the computation of the density of liquid in a silica hydrophilic nanopore was executed by the grand canonical Monte Carlo (GCMC) simulation to investigate the spatial dielectric properties of water in a confined phase. We found that the average values of the dielectric constants were very close and almost independent of the number of concentric radial shells. In response to the comment by $\mathrm{S}$. Mondal and B. Bagchi, we clarify the issues of reproducibility of bulk value of the dielectric constant of water and dielectric anisotropy.

\section{Introduction}

We published recently in $R S C$ Advances a paper, ${ }^{1}$ reporting the dielectric properties of pure water confined in a silica hydrophilic nanopore determined from the computation of the density of liquid in the confined phase by grand canonical Monte Carlo (GCMC) simulations. In this work, the dielectric constant of pure water was found to be reduced by a factor of more than 2 with respect to its bulk value (the average $\varepsilon_{\mathrm{p} \text {,water }} / \varepsilon_{\mathrm{b} \text {,water }}$ ratio was found to be 0.44$)$. That was imputed to water molecules located within the first three shells in the vicinity of the pore surface which exhibit preferential orientation with $\theta \sim 90$ to $100^{\circ}$ in nanoconfined phase.

In the preceding comment, S. Mondal and B. Bagchi raised two issues regarding our findings. (i) They declared that our calculation cannot reproduce the bulk value of the dielectric constant of water and (ii) we neglected the inherent anisotropy and non-local nature of dielectric response under confinement. This Reply aims to respond to both these points.

\section{Discussion}

First, S. Mondal and B. Bagchi performed the atomistic molecular dynamics simulations of confined SPC/E water inside carbon nanotubes. However, we carried out our molecular dynamics simulations with the most populated MCM-41 matrix of a silica hydrophilic nanopore and TIP4P-2005 water model.

${ }^{a}$ State Key Laboratory of Pollution Control and Resources Reuse, Key Laboratory of Yangtze River Water Environment, Ministry of Education, College of Environmental Science and Engineering, Tongji University, 1239 Siping Rd., Shanghai 200092, China.E-mail: ligm@tongji.edu.cn

${ }^{b}$ Shanghai Institute of Pollution Control and Ecological Security, Shanghai 200092, P. R. China
The different geometries and water models considered in their work and in the present work make difficult a direct comparison between our results. Secondly, they claimed that our calculation cannot reproduce the bulk value of the dielectric constant of confined water. This point is very confusing because it is currently believed that confinement should decrease dielectric constant since solvent molecules in such environments are expected to exhibit a greater degree of spatial and orientational order, especially in a few nanometers of space. Although some nanoscale applications like nanofiltration (NF) were found to lead to contradictory results, predicting either a decrease or an increase in the dielectric constant of the solution confined inside pores with respect to its bulk value,,$^{2-5}$ no experimental measurement of the dielectric constant inside pores of NF membranes have been reported yet. Therefore, our original paper is of great value for the calculation of the dielectric constant of water in a confined system. On the other hand, our simulation system is completely different from the one used in the comment as we have mentioned above.

S. Mondal and B. Bagchi also claimed that we neglected the inherent anisotropy and non-local nature of dielectric response under confinement. It is true that $\varepsilon$ is a tensor and exhibits a strong dependence on the shape and the size of the enclosure in a confined phase. Thus, dielectric behavior of water become anisotropic in nature and exhibit a diverse range of anomalies. In fact, we are not ignoring this point. Since our work focused on the variation of the permittivity in different areas of the nanopores, we presented the value of the average permittivity and did not discuss the permittivity in axial and radial directions separately, which would make our study more complicated. The following is our calculation method of the dielectric constant of water in a confined phase. 
As suggested by Lin and coworker, eqn (10) in our paper can be also used to compute the dielectric permittivity of the confined water and solution. ${ }^{6}$ The change in polarization $(\Delta \mathbf{P}(r))$ is determined from the linearized version of the fluctuationdissipation dielectric function given by as follow,

$$
\Delta \mathbf{P}(r)=\beta\left[\langle\mathbf{P}(r) \mathbf{M}\rangle_{0}-\langle\mathbf{P}(r)\rangle_{0}\langle\mathbf{M}\rangle_{0}\right] \mathbf{F}
$$

where $\beta$ is the inverse thermal energy, $\mathbf{F}$ is an external homogeneous electrical field and $\langle\cdots\rangle_{0}$ denotes ensemble averages in absence of $\mathbf{F}$.

In a cylindrical geometry, the confined medium is only inhomogeneous in $z$ direction and thus the local diagonal tensor can be defined by both parallel $\left(\varepsilon^{\|}\right)$and orthogonal $\left(\varepsilon^{\perp}\right)$ to the walls

$$
[\varepsilon]=\left(\begin{array}{lll}
\left(\varepsilon^{\|}(z)\right) & & \\
& \left(\varepsilon^{\|}(z)\right) & \\
& & \left(\varepsilon^{\perp}(z)\right)
\end{array}\right)
$$

As in the cylindrical case, the parallel dielectric lead to eqn (3),

$$
\varepsilon^{\|}(z)-1=\frac{\Delta \mathbf{P}^{\|}(z)}{\varepsilon_{0} \Delta \mathbf{E}^{\|}}
$$

where $\varepsilon_{0}$ the vacuum permittivity and $\Delta \mathbf{E}^{\|}$is the change of electric field that related to a local change in displacement field D. According to Maxwell's equation, $\Delta \mathbf{E}^{\|}=\mathbf{E}^{\|}$, which implies the average tangential electric field is constant. The parallel filed $\mathbf{F}^{\|}$ in eqn (1) refers to $\mathbf{E}^{\|}$. Eqn (3) thus can be rearranged with considering eqn (1),

$$
\varepsilon^{\|}(z)-1=\varepsilon_{0}{ }^{-1} \beta\left[\left\langle\mathbf{P}^{\|}(z) \mathbf{M}^{\|}\right\rangle_{0}-\left\langle\mathbf{P}^{\|}(z)\right\rangle_{0}\left\langle\mathbf{M}^{\|}\right\rangle_{0}\right]
$$

For orthogonal dielectric, perpendicular displacement field is constant on average, $\Delta \mathbf{D}^{\perp}(z)=\mathbf{D}^{\perp}$, and the perpendicular dielectric can be expressed as follow,

$$
1-\frac{1}{\varepsilon^{\perp}(z)}=\frac{\Delta \mathbf{P}^{\perp}(z)}{\mathbf{D}^{\perp}}
$$

The homogeneous field $\mathbf{F}^{\perp}$ corresponds to $\mathbf{D}^{\perp} / \varepsilon_{0}$ in orthogonal case. Therefore, combining eqn (1) and (5) gives,

$$
1-\frac{1}{\varepsilon^{\perp}(z)}=\frac{\beta\left[\left\langle\mathbf{P}^{\perp}(z) \mathbf{M}^{\perp}\right\rangle_{0}-\left\langle\mathbf{P}^{\perp}(z)\right\rangle_{0}\left\langle\mathbf{M}^{\perp}\right\rangle_{0}\right]}{\varepsilon_{0}}
$$

Note that the eqn (4) and (6) are the suitable undulation formulas to calculate the dielectric tensor in one direction of a nonhomogeneous system. These formulas depend only implicitly on the calculation of dielectric constant in confined phase. $^{7,8}$

Thus, we deal with two different kinds of polarization fluctuations leading to two different dielectric constants in a given coordinate system. The overall average static dielectric constant also gets defined in two ways, as shown in eqn (7),

$$
\varepsilon_{\mathrm{avg}}=\frac{\varepsilon^{\|}(z)+2 \varepsilon^{\perp}(z)}{3}
$$

\section{Conclusion}

In this reply article we have responded to the comments of S. Mondal and B. Bagchi. We consider that the different geometries and water models considered in their work and in our original work make difficult a direct comparison between our results. In addition, we acknowledge S. Mondal and B. Bagchi for their comments on the anisotropy of the dielectric constant of water in a confined system. Since our work focused on the variation of the permittivity in different areas of the nanopores, we did not discuss the permittivity in axial and radial directions separately. We recognize thus the limitations of the calculations in the originally published work and further work on this point needs to be taken seriously.

\section{Conflicts of interest}

There are no conflicts to declare.

\section{Acknowledgements}

This work was supported by Natural Science Foundation of Shanghai (20ZR1462900), National Natural Science Foundation of China (21603164), Major Science and Technology Program for Water Pollution Control and Treatment (2017ZX07207004) and Shanghai Science and Technology Innovation Action Plan (19DZ1204304).

\section{References}

1 H. Zhu, F. Yang, Y. Zhu, A. Li, W. He, J. Huang and G. Li, RSC Adv., 2020, 10, 8628-8635.

2 A. Szymczyk and P. Fievet, J. Membr. Sci., 2005, 252, 77-88.

3 S. Bouranene, A. Szymczyk, P. Fievet and A. Vidonne, Desalination, 2009, 240, 94-98.

4 S. Déon, P. Dutournié and P. Bourseau, AIChE J., 2007, 53, 1952-1969.

5 V. Silva, Dr. Ing. Thesis in Facultad de Ciencias, Universidad de Valladolid, Valladolid, 2009, p. 208.

6 Y. Lin, J. Shiomi, S. Maruyama and G. Amberg, Phys. Rev. B: Condens. Matter Mater. Phys., 2009, 80, 045419.

7 V. Ballenegger and J.-P. Hansen, J. Chem. Phys., 2005, 122, 114711.

8 D. J. Bonthuis, S. Gekle and R. R. Netz, Phys. Rev. Lett., 2011, 107, 166102. 